\title{
Comparative Study of Radio Models for data Gathering in Wireless Sensor Network
}

\author{
Joydeep Banerjee \\ Undergraduate Student \\ Advanced Digital and \\ Embedded Systems \\ Laboratory \\ Jadavpur University \\ Kolkata, India
}

\author{
Swarup Kumar Mitra \\ Department of ECE \\ M.C.K.V.I.E, LiLuah, Howrah \\ Kolkata, India
}

\author{
Mrinal Kanti naskar \\ Professor \\ Advanced Digital and \\ Embedded Systems \\ Laboratory \\ Jadavpur University \\ Kolkata, India
}

\begin{abstract}
Wireless Sensor Network (WSN) consists of irreplaceable nodes which are equipped with limited energy resources. Necessity of power consumption becomes a prior importance for various pervasive and ubiquitous applications. For realistic computation of energy in accordance with available motes like Micaz, Telos, Mica2, a discrete radio model exist. In this paper we have incorporated a discrete radio model over popular data gathering algorithm. We have formulated a data sheet, which relates details of power required to transmit data packets over range of distance that includes Lognormal Shadowing model for acquiring the required received signal strength. Range of distance is the key factor for energy consumption, which justifies the reduction of power levels to a limited count. We have disseminated the power transmission into specific band with respect to distance. Our paper shows the variation of node deployment over Network lifetime, which produces a significant alteration from sparse to dense network. We have conducted a comparison of Network lifetime and Mean energy consumption for chain based, shortest-hop and load balanced energy aware routing protocols. We have conducted a comparative study of the proposed method in TinyOS platform while running the simulation in TOSSIM.
\end{abstract}

\section{General Terms}

Wireless Sensor Network, Power dissipation Model.

\section{Keywords}

Micaz, Discrete Radio Model, Log Normal Shadowing Model, Energy Bands, Network Lifetime, TOSSIM.

\section{INTRODUCTION}

Wireless Sensor Network (WSN) consists of several nodes in count of hundred or thousand operating in remote location and harsh environment. Recent advancements in the field of digital signal processors, short-range radio electronics, microelectromechanical systems (MEMS) based sensor technology and low power RF designs have enabled the development of inexpensive low power sensors with significant computational capability.
Applications of sensor networks vary widely from climatic data gathering, seismic and acoustic underwater monitoring to surveillance and national security, military and health care. The major resource constraint is the energy consumption in the network as the sensor nodes being equipped by irreplaceable batteries. A network with even topology is deployed randomly with sensor nodes. The sensor networks are required to transmit gathered data to the base station (BS) or sink. Each node is provided with transmit power control and omni directional antenna and therefore can vary the areas of its coverage Since communication requires significant amount of energy compared to computations, sensor nodes must collaborate in an energyefficient manner for transmitting and receiving data so that lifetime can be enhanced and also a better "energy versus delay" [1] performance is achieved. The real issue of energy balancing in WSN can be utilized fully through an efficient power control of nodes. In this paper we have implemented different data gathering protocols using discrete power control [2]. We introduce a radio model, as discussed in [2], which dynamically determines which power level setting should be used to transmit between two nodes. Using the power level setting, the cost of transmission is calculated based on the Chipcon CC2420 radio chip [3] specifications to ensure an accurate estimation.

The novelty of our paper is that for realistic implementation of routing algorithms we have taken into consideration of seven discrete power levels as in Crossbow Micaz motes [3]. Secondly we assume Log-normal Shadowing path loss model, which detects an occurrence of an event at a particular distance from the node [4], for calculation of received signal strength (RSS). We have performed an analysis of the discrete model and CC2420 data sheet for determination of various power levels in term of distance and calculated the energy required to transmit data packets to a distance. For performing the above analysis we have taken the help of the experimentation and simulations that we made to calculate the discrete power in terms of distance. We have also made a chart for power output of all the available 32 
power levels and used the same in our calculations. We have applied our realistic power consumption model over the most popular energy efficient routing protocols which include shortest hop (SHORT) [5], chain formation (PEGASIS) [6] and load balancing (LBEERA) [7] data gathering algorithm.

The remainder of the paper is organized as follows. Section 2 describes the Network radio model and the radio propagation path loss model. Section 3 deals with Data Gathering Realistic Power Control. Simulation results are included in section 4 and finally Section 5 concludes the paper.

\section{NETWORK RADIO MODEL}

A typical sensor node consists of four major components: a data processor unit, a micro-sensor unit, a radio communication subsystem that consisting of transmitter/ receiver electronics, antenna, and amplifier; and a power supply unit. Although energy is dissipated by the first three components of a sensor node, we mainly consider the energy dissipation associated with the radio component.

\subsubsection{First Order Radio Model}

We consider the first order radio model as discussed in with identical parameter values. The energy per bit spent in transmission is given by

$$
e_{t x}(d)=e_{t}+e_{d} * d^{n}
$$

where $e_{t}$ is the energy dissipated per bit in the transmitter circuitry and $e_{d} * d^{n}$ is the energy dissipated for transmission of a single bit over a distance $\mathrm{d}$, $\mathrm{n}$ being the path loss exponent (usually $2.0 \leq n \leq 4.0$ ). For a first order model we assume $n=2$ for simulation purposes.

Thus the total energy dissipated for transmitting a K-bit packet is

$$
\mathrm{E}_{\mathrm{tx}}(\mathrm{K}, \mathrm{d})=\left(\mathrm{e}_{\mathrm{t}}+\mathrm{e}_{\mathrm{d}} * \mathrm{~d}^{2}\right) * \mathrm{~K}
$$

If $e_{r}$ be the energy required per bit for successful reception then the energy dissipated for receiving a K-bit packet is

$$
\mathrm{E}_{\mathrm{rx}}(\mathrm{K})=\mathrm{e}_{\mathrm{r}} * \mathrm{~K}
$$

In our simulations we take $e_{t}=50 \mathrm{~nJ} / \mathrm{bit}, \mathrm{e}_{\mathrm{d}}=100 \mathrm{pJ} / \mathrm{bit} / \mathrm{m}^{2}$ and $e_{r}=e_{t}$ as mentioned in [5] with $\mathrm{K}=2000$ bits. It is assumed that the channel is symmetric so that the energy spent in transmitting from node $i$ to $j$ is the same as that of transmitting from node $j$ to i.

\subsection{Discrete Radio Model}

In previous works, authors assume that the power level can be adjusted to the exact need and calculate the energy cost using these exact values. In reality this is not the case as the radio can only be adjusted to one of the associated power levels and not set to the exact transmission power needed. Using the assumption that there is an infinite amount of transmission levels, previous work makes the assumption that the longer links will cost more to transmit a packet. In many situations two links of different lengths will need to transmit at the same power level setting in order for the packet to be received and therefore the cost of transmission over different distances can be equivalent. We also have analyzed the basic parameters involved in the conventional first order radio model and discrete radio model. The power level consideration from the actual data sheet of Micaz [3] is also discussed in relevant sections. The sensing of an event depends also on the received signal strength indication (RSSI).

In this paper we introduce a radio model, which dynamically determines the power level setting should be used to transmit between two nodes. Using the power level setting, the network lifetime and mean energy of the network is calculated based on the chip specifications to ensure an accurate estimation of lifetime. Transmission can only occur at predefined discrete power levels of the amplifier, based on the RSSI [2] value needed for communication. Discrete radio model provides more realistic simulations. However in real world deployments there may be obstacles and environmental conditions that prevent accurate estimation. Due to this, the estimated power levels for transmission between nodes can only be used as a starting point. Energy dissipation for transmission is constant for a particular power level whereas energy required to receive a data is same for all power levels

\subsection{Realistic Power Data Gathering Algorithm}

The first order radio model has certain drawbacks. There is a calculated energy change for a small change in distance of communication, as it recognizes the transmit power to depend upon distance in an analogous manner. So we can argue, according to the equation of transmit power for first order radio model, that there is also a calculated power to which a node can operate to send data beyond its limit of distance. Moreover it is developed taking into consideration of fading of the transmitted signal that may take place due to the environmental conditions. The discrete radio models also did not incorporate such considerations and as discussed the power out at various power levels can only be used at the starting point. We have implemented log normal shadowing model as the fading model.

\subsubsection{Log Normal Shadowing Model}

In probability theory, a log-normal distribution is a probability distribution of a random variable whose logarithm is normally distributed. If $Y$ is a random variable with a normal distribution, then $X=\exp (Y)$ has a $\log$-normal distribution; likewise, if $X$ is $\log$-normally distributed, then $Y=\log (X)$ is normally distributed. (This is true regardless of the base of the logarithmic function: if $\log _{a}(Y)$ is normally distributed, then so is $\log _{b}(Y)$, for any two positive numbers $a, b \neq 1$.)

Sensing is a probabilistic feature which introduces a signal loss component which depends on antenna height of the transmitter and receiver as well as distance between transmitting and receiving mote. For obtaining the actual received signal strength we have introduced a Log normal shadow model as shown in Figure 1. Figure 1 shows how the initial input changes and attenuates to a certain low stabilized value. The concept of fast 
and slow fading of the signals is also described. The corresponding value of slow fading for a full range of fast fading and the corresponding effect of a full range slow fading in the reduction of Received Signal Strength (RSS) is also shown. This is the best representation of that for power output of the sensor motes for signal calculations at different points. The dependencies of all the factors (obstacles such as building, foliage etc.) have been taken into account in this sensing model and have been implemented as a memory less probability distribution function. The sensing ability of a mote is not uniform in all directions hence we have assumed a log normal shadow fading sensing model to calculate the signal loss. The signal loss for the plane earth model [4] is given by equation 1 .

$\mathrm{L}(\mathrm{d})=\mathrm{L}\left(\mathrm{d}_{0}\right)+10 * \beta * \log \left(\mathrm{d} / \mathrm{d}_{0}\right)$

Where $\beta=$ is the path loss exponent and $L\left(d_{0}\right)$ is the path loss measured at distance ' $\mathrm{d}_{0}$ '.

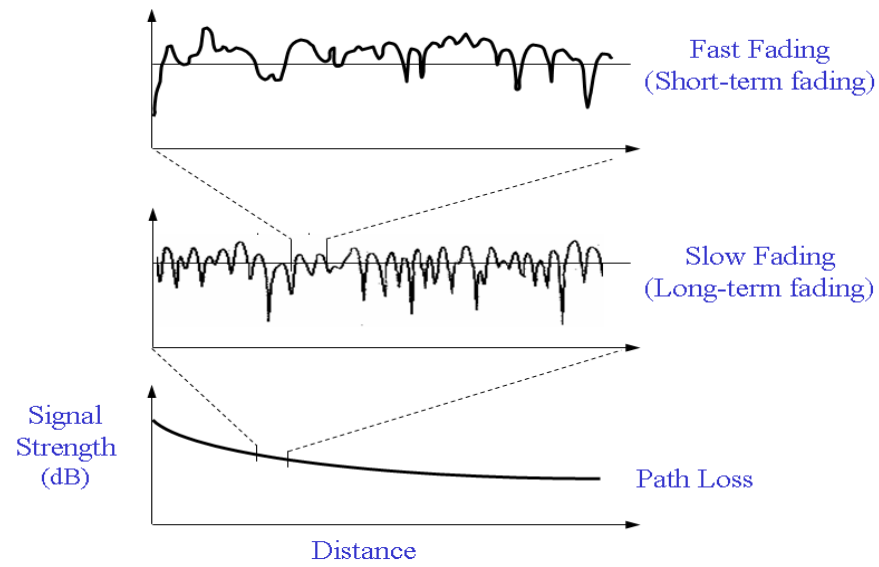

Figure1. Effect of fading on Received Signal Strength according to log normal shadowing model.

\section{REALISTIC POWER MODEL}

The discrete radio model [2] is a technique for balancing the power levels of the network irrespective of distance and node failures. The discrete model uses 32 distinct power levels for CC2420 trans-receiver. The power levels and their corresponding power output (in $\mathrm{dbm}$ ) are enumerated below in Table I.

The values in Table I were calculated by interpolation method. But due to close proximity in the range in terms of sensing distance and the power output some of the power levels are never used. More over simulation in Tiny Os leads to the conclusion that if a mode is set at power level 6, power is transferred itself to mode. Similarly for each power level the motes gets switched over to the nearest power level among 3, 7, $11,15,19,23,27$ and 31. So rests of the power levels are discarded from the discussion and only the above mentioned relevant power levels are taken into consideration.
Nodes have packets of data in each round of communication that need to be gathered and fused with packets of other nodes packet into one packet and get transmitted to distant Base Station. We have introduced a radio model that dynamically determines the power level setting that should be used to transmit between two nodes. For discrete radio model setting the transmit power and the received power are given in equations 5 and 6 respectively.

Table I. Power outputs for the discrete power levels available.

\begin{tabular}{|l|l|l|l|l|l|}
\hline $\begin{array}{l}\text { Power } \\
\text { Level }\end{array}$ & $\begin{array}{l}\text { Power } \\
\text { Output } \\
\mathbf{d B m}\end{array}$ & $\begin{array}{l}\text { Power } \\
\text { Level }\end{array}$ & $\begin{array}{l}\text { Power } \\
\text { Output } \\
\mathbf{d B m}\end{array}$ & $\begin{array}{l}\text { Power } \\
\text { Level }\end{array}$ & $\begin{array}{l}\text { Power } \\
\text { Output } \\
\mathbf{d B m}\end{array}$ \\
\hline 0 & -37.90 & 11 & -10.00 & 22 & -3.520 \\
\hline 1 & -33.00 & 12 & -9.120 & 23 & -3.000 \\
\hline 2 & -28.70 & 13 & -8.330 & 24 & -2.470 \\
\hline 3 & -25.00 & 14 & -7.630 & 25 & -1.950 \\
\hline 4 & -21.80 & 15 & -7.000 & 26 & -1.450 \\
\hline 5 & -19.20 & 16 & -6.440 & 27 & -1.000 \\
\hline 6 & -16.90 & 17 & -5.940 & 28 & -0.610 \\
\hline 7 & -15.00 & 18 & -5.470 & 29 & -0.310 \\
\hline 8 & -13.40 & 19 & -5.000 & 30 & -0.091 \\
\hline 9 & -12.10 & 20 & -4.520 & 31 & 0 \\
\hline 10 & -11.00 & 21 & -4.030 & - & - \\
\hline
\end{tabular}

$\mathrm{P}_{\mathrm{TR}}=\mathrm{V}_{\text {REMAINING }} *\left(\mathrm{I}_{\mathrm{ROON}} * \tau+\mathrm{I}_{\mathrm{PLEVEL}} *(\mathrm{~L} / \mathrm{R})\right)$

$\mathrm{P}_{\mathrm{RE}}=\mathrm{V}_{\text {REMAINING }} * \mathrm{I}_{\mathrm{R}} *(\mathrm{~L} / \mathrm{R})$

Where, $\mathrm{V}_{\text {REMAINING }}=$ remaining current voltage of the mote, $\mathrm{I}_{\mathrm{ROON}}=$ Current required for radio oscillator to start, $\tau=$ oscillator start up time and is equal to $0.86 \mathrm{~ms}, \mathrm{I}_{\mathrm{PLEVEL}}=$ current at a power level (shown in TABLE II, discussed later). $\mathrm{L}=$ packet size ( 1 packet is taken to be as 100 bits), $\mathrm{R}=$ rate of transmission and is equal to 256 kilo bits per second, $I_{R}=$ current required to receive data and is equal to $19.60 \mathrm{~mA}$. The above specifications are supplied as per CC2420 data sheet of [3].

Now we have made a study of the various power levels output with distance. We have incorporated the fading model according to $\log$ normal shadowing model. The power in $\mathrm{dBm}$ for various power levels with their progressive decrease in power with distance is shown in Figure 2. The CC2420 trans-receiver, as in [3] can sense data appropriately to a lowest received power of $90 \mathrm{dBm}$. From Figure 2 we can compute the distance or range of distance in which various power levels operate. The range of distance is calculated by taking into consideration the lowest power level for that range. We have tabulated the distance range for various power levels, the energy required to transmit a bit of data at that power level and the corresponding current consumed in Table II. In Table III we provide the same data except the distance range (as there is no distance range for receiving). We 
have also eradicated the power level 15 due to its closeness of distance range with power level 11 and 19.

Discrete order Radio Model operates on the principle of Energy Bands and it works irrespective of distance and number of packets. Traditional radio model mainly focuses on the distance of sensor nodes from Base Station (BS) together with the count of number of hops during transmission, i.e. PEGASIS [5] and also for shortest hop between each communication pair of nodes ,i.e. SHORT [6]. It also depends on the number of packets. In reality a transmit power level of the sensor node can only be adjusted to discrete values which may result in single power level for multiple values of distance. We have applied realistic power consumption model over chain based protocol PEGASIS [5] and in shortest hop algorithm (SHORT) [6]. For further analysis we have also applied the technique in load balancing of energy for leader and super leader selection i.e. LBEERA [7] where the network is divided into 5 equal partitions in terms of area and chain based routing is applied to each partition with leader of each partisan exhibiting a similar kind of routing and the final message is sent to the base station via a super leader selected among the leader.

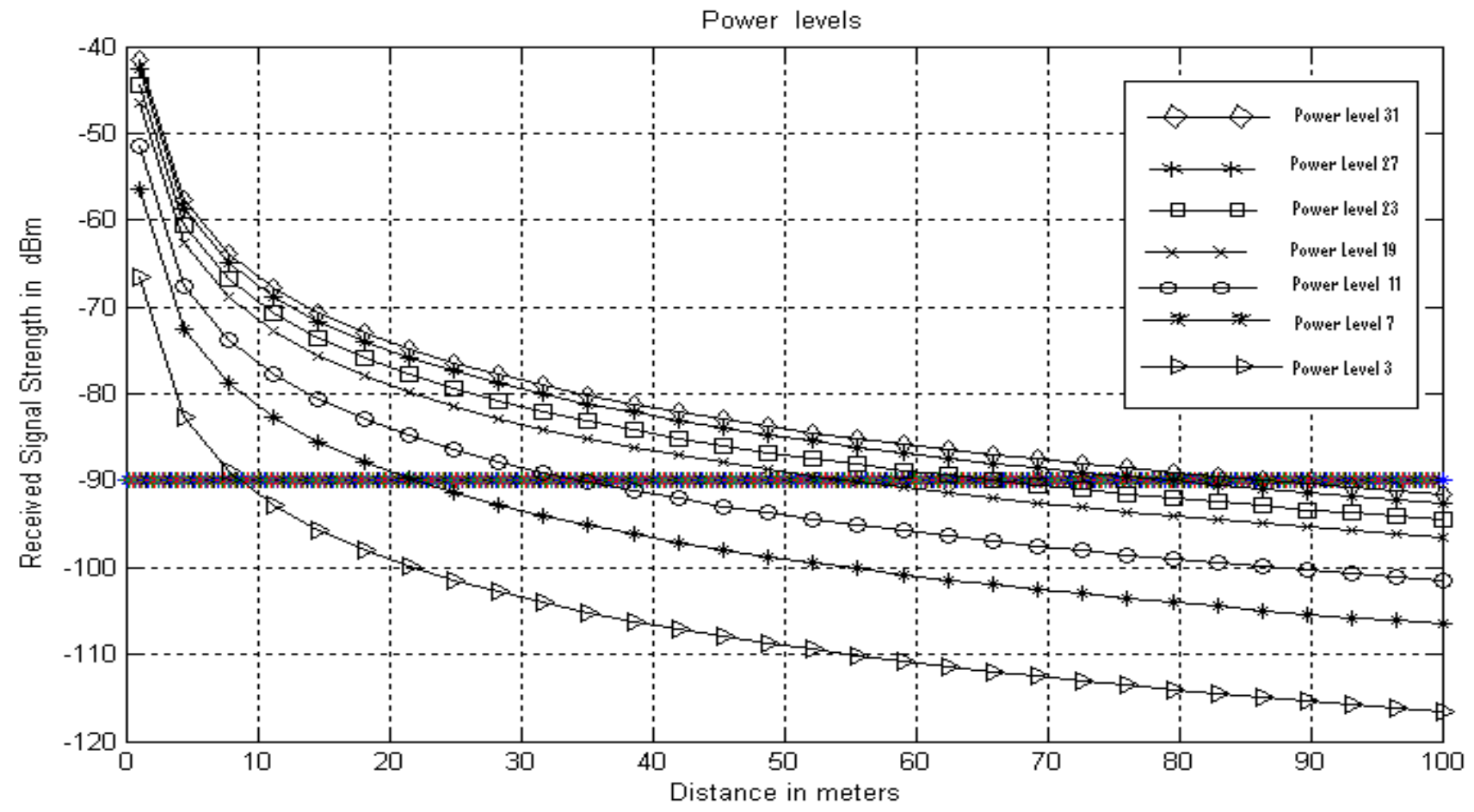

Figure 2. Received Signal Strength in $\mathbf{d B m}$ vs. distance (for seven mentioned discrete power levels)

Due to this, the estimated power levels for transmission between nodes can only be used as a starting point. The transmit and receive power level is selected from the Table I and II which provides energy consumed in transmission of 100 bit packet considering the different power levels used by CC2420.We have neglected power level 15 as it has a power output of $-7 \mathrm{dbm}$ and simulation yields that the distance of operation of this power level is so close to power levels 11 and 19 such that it becomes meaningless to take this power level in the context of number of packets of energy sent in the simulation. The number of packets sent in one transmission is taken to be as 20 . So a total of 2000 bits are sent.

As most data gathering algorithms are controlled by the centralized base station configuration, we will draw our attention mostly on these types of networks. Discrete power level selection produces a major advantage on these networks. Firstly, only the base station needs to track and store the node which tries to communicate at its desired power level. This generates free memory and storage space which is limited on each mote, allowing more data gathering to occur or storing more data before transmitting it back to the base station. Secondly, with a centralized configuration the base station will know the exact power levels of each mote which we are using for transmission and therefore the exact power cost. In order to select the best power levels in a real world deployment we propose that a network initialization period is used. In this period, each mote takes a turn and broadcasts a packet at each power level with the assigned mote ID and the transmission power level being used. Each other mote in the network will listen for the packet and track the lowest power that can be used for communication from that mote. Once every mote has participated in the initialization period, they generate a packet containing their mote ID and an array of incoming packet information. This incoming packet information will contain the incoming mote ID, lowest received power level and the associated RSSI. and the base station can dictate which power levels should be 
Table II. Power output, distance of operation, current, and transmitted energy per bit for various power levels.

\begin{tabular}{|c|c|c|c|c|c|}
\hline $\begin{array}{c}\text { Power } \\
\text { level }(\mathbf{k})\end{array}$ & $\underset{[\mathbf{d B m}]}{\mathbf{P}_{\text {out }}}$ & $\begin{array}{l}\text { Distance (in } \\
\text { meters) }\end{array}$ & $\mathbf{I}_{\mathbf{x}}(\mathbf{m A})$ & $\underset{(\mathbf{m W})}{\mathbf{P}_{\mathrm{TX}}}$ & $\begin{array}{l}\mathbf{E}_{\text {tx }} / \text { bit } \\
{[\mu J]}\end{array}$ \\
\hline 3 & $\begin{array}{c}- \\
25.00\end{array}$ & $\mathrm{~d}<8.7 \mathrm{~m}$ & 17.04 & 15.15 & 0.0606 \\
\hline 7 & - & $8.7 \mathrm{~m}<\mathrm{d}<21.86$ & 15.78 & 17.47 & 0.0699 \\
\hline 11 & $\begin{array}{c}- \\
10.00\end{array}$ & $\begin{array}{l}21.86<\mathrm{d}<34.6 \\
1\end{array}$ & 14.63 & 19.62 & 0.0785 \\
\hline 19 & -5.00 & $\begin{array}{l}34.61<\mathrm{d}<54.9 \\
7\end{array}$ & 12.27 & 22.08 & 0.0883 \\
\hline 23 & -3.00 & $\begin{array}{l}54.97<\mathrm{d}<62.2 \\
2\end{array}$ & 10.91 & 26.33 & 0.1050 \\
\hline 27 & -1.00 & $\begin{array}{l}62.22<d<71.6 \\
7\end{array}$ & 9.71 & 28.40 & 0.1136 \\
\hline 31 & 0 & $74.47<\mathrm{d}<81$ & 8.42 & 30.67 & 0.1227 \\
\hline
\end{tabular}

Table III. The value of current, power dissipated and the energy received per bit

\begin{tabular}{|c|c|c|c|}
\hline $\mathbf{I x}[\mathbf{m A}]$ & $\mathbf{P}_{\mathbf{R x}}($ in $\mathbf{m W})$ & $\mathbf{E}_{\mathbf{R x}} /$ packet & $\mathbf{E r x} / \mathbf{b i t}[\boldsymbol{\mu} \mathbf{J}]$ \\
\hline 19.60 & 35.28 & 112.90 & 0.1411 \\
\hline
\end{tabular}

This packet is transmitted to the base station for storage and processing, which relieves the mote from storing the information. With this information at the base station, the data gathering algorithms can be optimized for the exact costs used when it broadcasts schedules to motes in the network.

\section{SIMULATION RESULTS}

In this paper, we investigate the combination of several data gathering techniques into a single model that will be more realistic and useful for simulations. We have investigated the performance over chain formation, PEGASIS [5], shortest hop data gathering [6] and LBEERA.

From Table II we can see that the largest distance the CC2420 can receive is 81 meters and any possibility of communication can be feasible beyond this distance. As we have used the data sheet of CC2420 in our simulation we have considered this fact. Firstly, we simulated the model consisting of 5 motes in TinyOs 2 platform by using TOSSIM simulator. In the simulation we fixed the initial energy of each mote to be $50 \mathrm{~mJ}$ and deployed it in a 20 meter square field with base station at a distance of $30 \mathrm{~m}$ from the mid point of the nearest side of the square field. Next we made the same simulation in MATLAB using the same initialization and deployment of nodes using the proposed realistic power model. In both the simulation the senor directly sends the event data to the base station and no routing protocol is employed.

A total of 50 such simulations were made by changing the deployment of the motes and the field size i.e. for various values of node density [7] (defined as the number of nodes per unit area of the sensing field) range from 0.01 to 0.1 . The criteria for the first node death (FND) and last node death (LND) [8] of the sensor network are plotted.

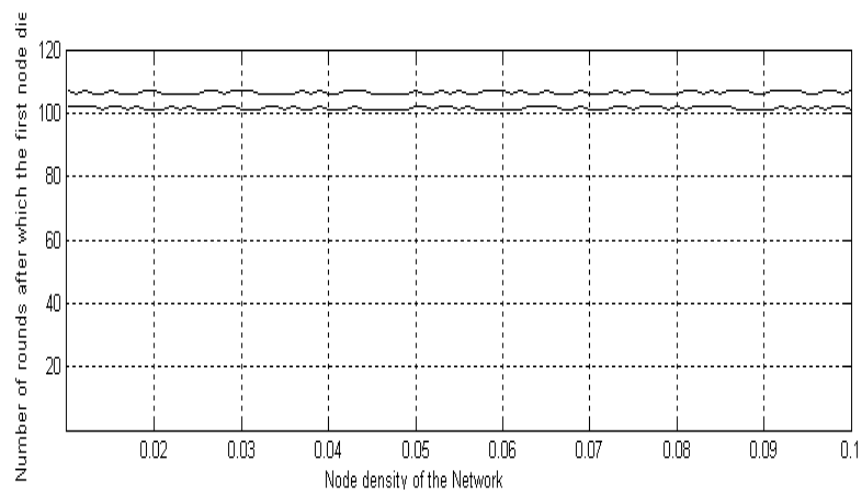

Figure 3: The plot of First Node Dies condition for actual power consumption and realistic power model with varying node density

Fig. 3 shows the criterion of FND for actual power dissipation and realistic power model. The realistic power model (which is the plot with greater value of number of round after which first node dies) closely follows the actual power dissipation (the lower plot is for realistic power model) with only $4.3 \%$ mean difference percentage between the two.

Figure 4 is the plot of the same but for the criterion of last node dies. Here we see that the real power model shows greater network lifetime in terms of LND than actual power consumption until 0.6 value of node density and then actual power consumption shows greater LND value. But altogether the mean difference percentage is only $2.95 \%$ which is significantly low. Hence the realistic power model proposed by us follows the actual power consumption and justifies its applicability as an efficient radio mode. 
Table IV. Network Lifetime under different radio models

\begin{tabular}{|c|c|c|c|c|c|c|c|}
\hline \multirow{3}{*}{$\begin{array}{c}\text { Data } \\
\text { Gathering } \\
\text { Scheme }\end{array}$} & \multirow{3}{*}{$\begin{array}{l}\text { Initial } \\
\text { Energ } \\
\mathrm{y}(\mathrm{mJ})\end{array}$} & \multicolumn{6}{|c|}{ Network Lifetime (No of rounds) } \\
\hline & & \multicolumn{3}{|c|}{ Discrete Radio Model } & \multicolumn{3}{|c|}{ First Order Radio Model } \\
\hline & & FND & $\begin{array}{c}10 \% \text { of } \\
\text { node } \\
\text { dies }\end{array}$ & LND & FND & $\begin{array}{c}10 \% \text { of } \\
\text { node } \\
\text { dies }\end{array}$ & $\begin{array}{l}\mathrm{LN} \\
\mathrm{D}\end{array}$ \\
\hline \multirow{3}{*}{$\begin{array}{l}\text { PEGASIS } \\
50 \mathrm{~m} \times 50 \mathrm{~m} \\
\text { square field } \\
\text { for } 100 \\
\text { sensors }\end{array}$} & 250 & 130 & 300 & 570 & 190 & 230 & 666 \\
\hline & 500 & 250 & 600 & 1164 & 400 & 625 & $\begin{array}{l}143 \\
4\end{array}$ \\
\hline & 1000 & 505 & 1250 & 2259 & 750 & 1180 & $\begin{array}{l}337 \\
1\end{array}$ \\
\hline \multirow{3}{*}{$\begin{array}{c}\text { PEGASIS } \\
100 \mathrm{~m} \times 100 \mathrm{~m} \\
\text { square field } \\
\text { for } 100 \\
\text { sensors }\end{array}$} & 250 & 205 & 295 & 541 & 75 & 110 & 599 \\
\hline & 500 & 400 & 580 & 1146 & 110 & 225 & $\begin{array}{l}132 \\
5\end{array}$ \\
\hline & 1000 & 520 & 1180 & 2254 & 250 & 520 & $\begin{array}{l}276 \\
9\end{array}$ \\
\hline \multirow{3}{*}{$\begin{array}{c}\text { SHORT } \\
50 \mathrm{~m} \times 50 \mathrm{~m} \\
\text { square field } \\
\text { for } 100 \\
\text { sensors }\end{array}$} & 250 & 232 & 260 & 330 & 540 & 570 & 550 \\
\hline & 500 & 430 & 520 & 630 & 1100 & 1150 & $\begin{array}{l}114 \\
5\end{array}$ \\
\hline & 1000 & 855 & 1005 & 1250 & 2210 & 2255 & $\begin{array}{l}237 \\
0\end{array}$ \\
\hline \multirow{3}{*}{$\begin{array}{c}\text { SHORT } \\
100 \mathrm{~m} \times 100 \mathrm{~m} \\
\text { square field } \\
\text { for } 100 \\
\text { sensors }\end{array}$} & 250 & 230 & 260 & 315 & 540 & 555 & 565 \\
\hline & 500 & 450 & 500 & 590 & 855 & 860 & 900 \\
\hline & 1000 & 850 & 980 & 1200 & 1750 & 1780 & $\begin{array}{l}179 \\
5\end{array}$ \\
\hline \multirow{3}{*}{$\begin{array}{l}\text { LBEERA } \\
50 \mathrm{~m} \times 50 \mathrm{~m} \\
\text { square field } \\
\text { for } 100 \\
\text { sensors }\end{array}$} & 250 & 125 & 170 & 413 & 280 & 310 & 566 \\
\hline & 500 & 250 & 340 & 810 & 560 & 610 & $\begin{array}{l}111 \\
0\end{array}$ \\
\hline & 1000 & 500 & 590 & 1620 & 900 & 1350 & $\begin{array}{l}222 \\
5\end{array}$ \\
\hline \multirow{4}{*}{$\begin{array}{c}\text { LBEERA } \\
100 \mathrm{~m} \times 100 \mathrm{~m} \\
\text { square field } \\
\text { for } 100 \\
\text { sensors }\end{array}$} & 250 & 390 & 650 & 1590 & 560 & 990 & 142 \\
\hline & & & & & & & \\
\hline & 500 & 97 & 175 & 380 & 82 & 210 & 360 \\
\hline & 1000 & 190 & 325 & 790 & 230 & 510 & 710 \\
\hline
\end{tabular}

After getting the suitability of our proposed radio model we made simulations in MATLAB to compare the first order radio model with the discrete radio model for PEGASIS and SHORT routing algorithms. The sensor networks we have taken under consideration for our simulation are

(i) We have considered here a square field of length 50 meter and 100 meter with base station located at $(25 \mathrm{~m}, 75 \mathrm{~m})$ and $(50 \mathrm{~m}, 150 \mathrm{~m})$ [9] respectively and deployed 100 sensor motes in that field. The base station is acknowledged to all the sensor nodes about its position which having enhanced computational capabilities with no energy constraints. The energy of the motes that we have considered for the simulation are $250 \mathrm{~mJ}, 500 \mathrm{~mJ}$ and $1000 \mathrm{~mJ}$.

(ii) Nodes are dispersed randomly following a uniform distribution in a 2-dimensional space which is static and stationary after deployment.

We have made 50 simulations and obtained the mean value of all output parameters for all the cases discussed above for better results and incorporation of realistic simulations. We have 
tabulated the rounds of communication when the first node dies (FND) and last node dies (LND) for each case in TABLE IV for PEGASIS, SHORT and LBEERA routing algorithm for all those cases. We have also compared the first order radio model and the realistic radio model in terms of number of alive nodes and the mean energy of the network in context of the number of rounds. Figure 5(a) and 5(b) gives the mean energy of the network in $\mathrm{mJ}$ and the number of alive nodes respectively with respect to number of rounds for both the radio models for PEGASIS with mean initial energy of network as $250 \mathrm{~mJ}$ and field length $50 \mathrm{~m} \times 50 \mathrm{~m}$. Figure $6(\mathrm{a})$ and $6(\mathrm{~b})$ gives the mean energy of the network in $\mathrm{mJ}$ and the number of alive nodes respectively with respect to number of rounds for both the radio models for SHORT with mean initial energy of network as 1000 $\mathrm{mJ}$ and field length $100 \mathrm{~m} \times 100 \mathrm{~m}$. Figure 7(a) and 7(b) gives the mean energy of the network in $\mathrm{mJ}$ and the number of alive nodes respectively with respect to number of rounds for both the radio models for LBEERA with mean initial energy of network as $2000 \mathrm{~mJ}$ and field length $100 \mathrm{~m} \times 100 \mathrm{~m}$.

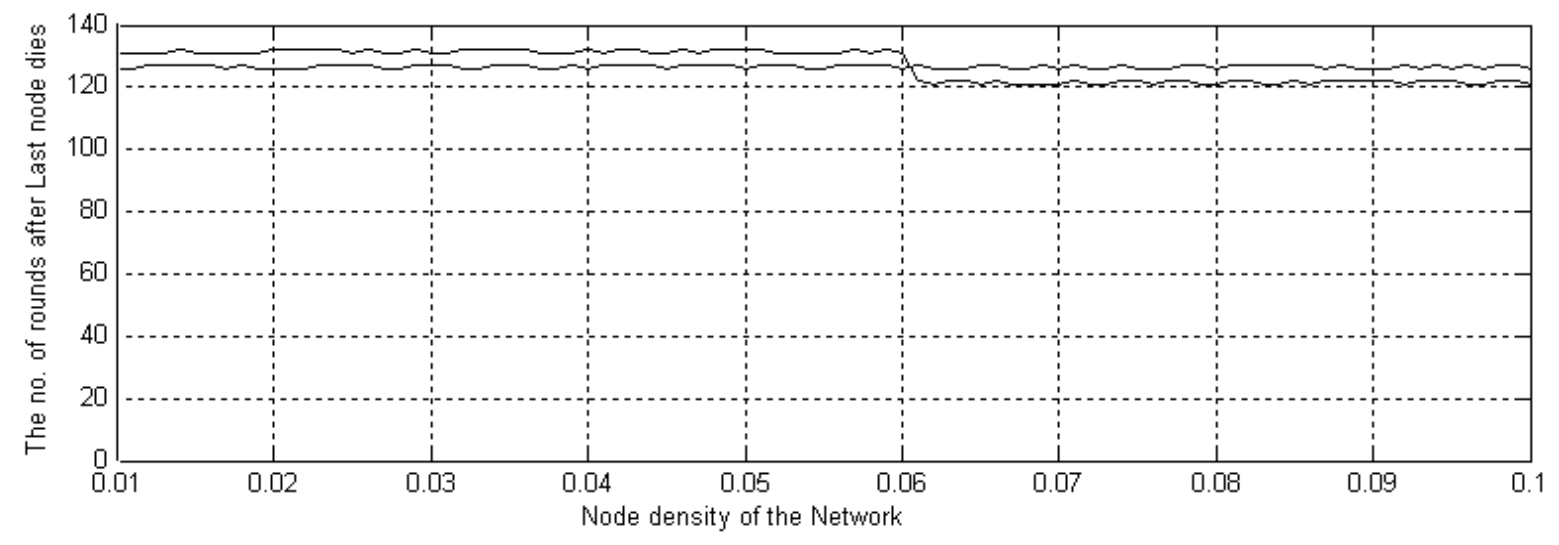

Figure 4: The plot of Last Node Dies condition for actual power consumption and realistic power model with varying node density

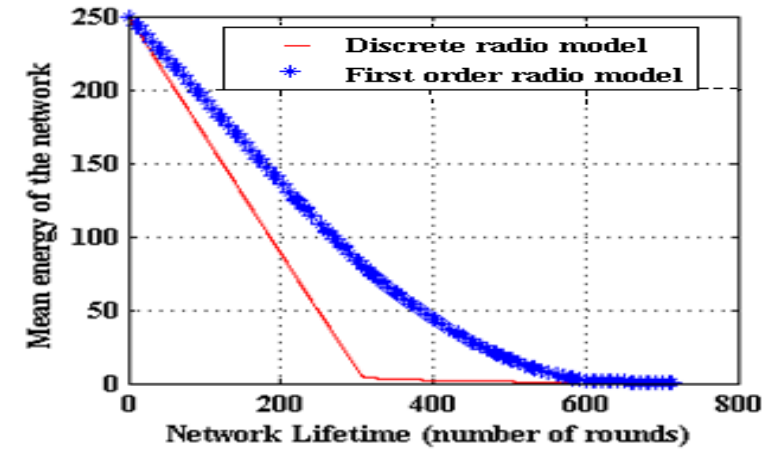

Figure 5(a). Comparison of radio models "Mean energy of the network" vs. "Network Lifetime" for a ( $50 \mathrm{X} 50)$ square field for 100 sensor nodes with initial energy of $250 \mathrm{~mJ}$ for PEGASIS

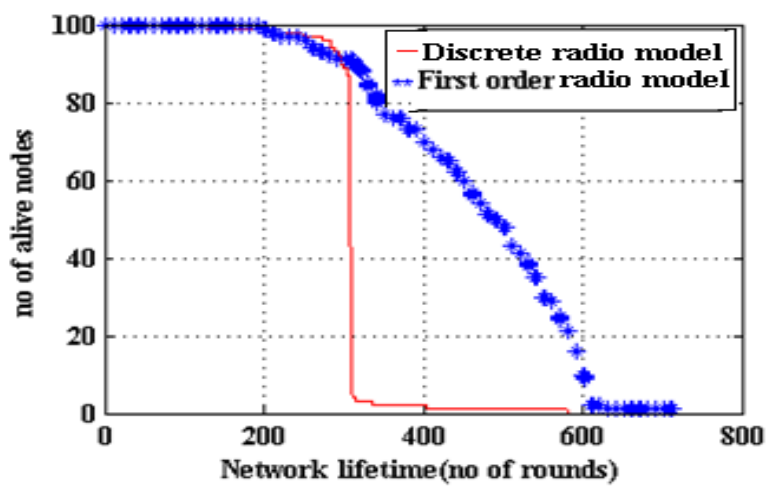

Figure 5 (b): Comparison of radio models "no of alive nodes" vs. "Network Lifetime" for a ( $50 \mathrm{~m}$ X 50m )square field for 100 sensor with initial energy of $250 \mathrm{~mJ}$ for PEGASIS 


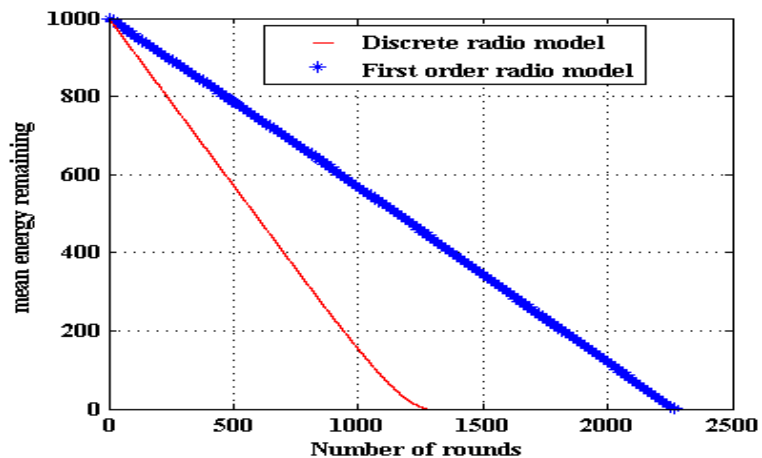

Figure 6(a) Comparison of radio models "Mean energy of the network" vs. "Network Lifetime" for a( $100 \mathrm{~m} X 100 \mathrm{~m})$ square field for 100 sensor nodes with initial energy of 1000 mJ for SHORT

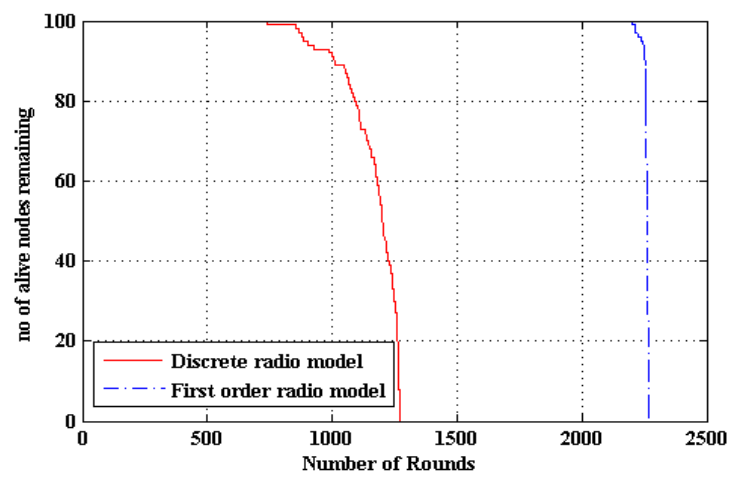

Figure 6(b). Comparison of radio models "no of alive nodes" vs. "Network Lifetime" for a(100m X $100 \mathrm{~m})$ square field for 100 sensor nodes with initial energy of $1000 \mathrm{~mJ}$ for SHORT

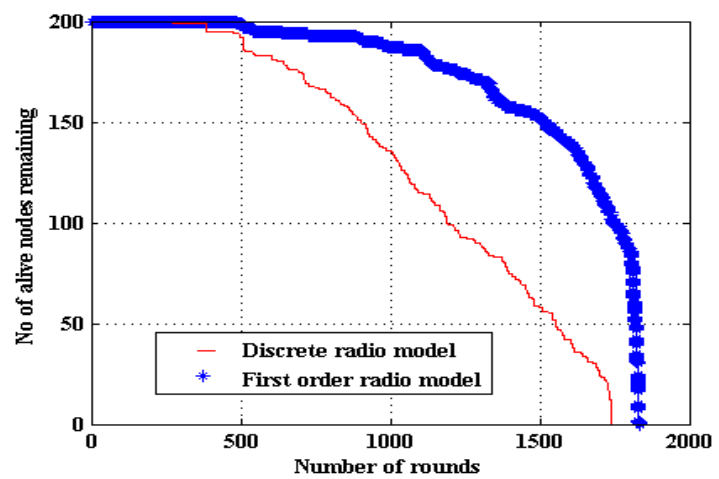

Fig 7 (a) No of alive nodes" vs "Network Lifetime" for a $(100 \mathrm{~m} \times 100 \mathrm{~m})$ square field for 200 sensors with initial energy $1000 \mathrm{~mJ}$ for LBEERA

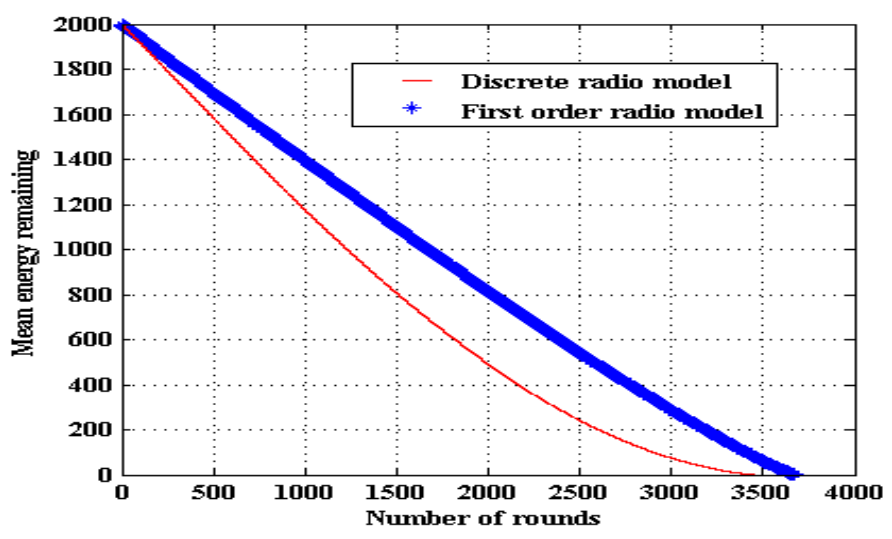

Fig 7 (b) Mean energy of the network" vs "Network Lifetime" for a $(100 \times 100)$ square field for 200 sensor nodes with initial energy of $2000 \mathrm{~mJ}$ for LBEERA

From the above simulation results it is justified that the first order radio model gives a large variation from the original power consumption model whereas the realistic power consumption model that we have assumed and proved in the first part of our simulation to closely follow the original power consumption.

The performance analysis and tabulated it in Table $\mathrm{V}$ which shows the network lifetime when the average energy of the network is less than $10 \mathrm{~mJ}$ for various routing algorithms. It shows that at the trailing end of the energy discrete radio model has a greater lifetime than first order radio model.

TABLE $V$ the network lifetime when the average energy of the network is less than $10 \mathrm{~mJ}$

\begin{tabular}{|c|c|c|c|}
\hline \multirow{2}{*}{$\begin{array}{l}\text { Data gathering } \\
\text { Scheme in a } \\
50 \mathrm{~m} \times 50 \mathrm{~m} \\
\text { square field for } \\
100 \text { sensors }\end{array}$} & \multirow{2}{*}{$\begin{array}{c}\text { Initi } \\
\text { al } \\
\text { Ener } \\
\text { gy }\end{array}$} & \multicolumn{2}{|c|}{$\begin{array}{l}\text { Network Lifetime ( no of rounds } \\
\text { )for remaining } 10 \mathrm{~mJ} \text { of Mean } \\
\text { energy of the Network }\end{array}$} \\
\hline & & $\begin{array}{c}\text { Discrete radio } \\
\text { Model }\end{array}$ & $\begin{array}{l}\text { First order radio } \\
\text { Model }\end{array}$ \\
\hline \multirow[t]{3}{*}{ PEGASIS } & 250 & 275 & 170 \\
\hline & 500 & 590 & 400 \\
\hline & 1000 & 850 & 600 \\
\hline \multirow[t]{3}{*}{ SHORT } & 250 & 30 & 10 \\
\hline & 500 & 35 & 20 \\
\hline & 1000 & 50 & 20 \\
\hline \multirow[t]{3}{*}{ LBEERA } & 250 & 30 & 40 \\
\hline & 500 & 40 & 30 \\
\hline & 1000 & 50 & 40 \\
\hline
\end{tabular}




\section{CONCLUSION}

In this paper, we have proposed an algorithm called Realistic Power Data Gathering Algorithm for collecting useful data from a remote wireless sensor network to the BS. The network lifetime and mean energy of the network is compared with realistic discrete radio model and first order radio model. Utility of the realistic radio model for node replacement and longer trailed lifetime is observed. But the overall the performance requirement is good in comparison with other algorithms. Our approach overcomes the losses incurred from all other data gathering schemes, compared with the network lifetime and utilization of the network. Moreover it follows the real power consumption phenomenon to a great extent. Altogether the realistic power model described by us is totally justified and can be applied to any data gathering scheme for their performance evaluation.

\section{REFERENCES}

[1] Lindsey S., Raghavendra C.S., and Sivalingam K., 'Data Gathering in Sensor Networks using energy*delay metric,' Proceedings of the $15^{\text {th }}$ International Parallel and Distributed Processing Symposium, (2001), pp.188-200

[2] Michael Mallinson, Patrick Drane, and Sajid Hussain., 'Discrete radio power level consumption model in wireless sensor networks,Second International Workshop on Information Fusion and Dissemination in Wireless Sensor Networks (Sensor Fusion), 2007

[3] C.T.Inc.,http://www.xbow.com/Products/Product_pdf_files /Wireless_pdf/MICAz_Datasheet.pdf.

[4] Asraf Hossain, P.K.Biswas, S.Chakrabarti, 'Sensing Model and Its Impact on Network Coverage in Wireless Sensor Network', proceedings of the $10^{\text {th }}$ Colloquium and The third ICIIS, Kharagpur, India 2008.

[5] Yang, Y., Wu, H.H., Chen, H.H., "SHORT: Shortest Hop Routing Tree for Wireless Sensor Networks", IEEE ICC 2006 proceedings, (2006).

[6] Lindsey, S.Raghavendra, C.S,"PEGASIS: Power Efficient Gathering in Sensor Information Systems," in Proceedings of IEEE ICC 2001 (2001) 1125-1130.

[7] Yu1, Y., Wei, and G.: Energy Aware Routing Algorithm Based on Layered Chain in Wireless Sensor Network, 14244-1312-5/07/\$25.00 @ 2007 IEEE.

[8] Ayon Chakraborty, Swarup Kumar Mitra, and M.K. Naskar, "An Efficient Hybrid Data Gathering Scheme in Wireless Sensor Networks", ICDCIT 2010, LNCS 5966, pp. 98-103, 2010. 